REVISIÓN DE LITERATURA

\title{
NITRÓGENO UREICO EN LECHE: IMPORTANCIA, DETERMINACIÓN Y RELACIÓN CON OTROS COMPONENTES LÁCTEOS
}

Jeffry Sánchez-Salas ${ }^{1}$

\section{RESUMEN}

La concentración de urea en leche es proporcional a la cantidad de urea en la sangre. Por esta razón, el análisis de nitrógeno ureico en leche (MUN) ha sido adoptado como herramienta para monitorear la eficiencia de utilización de nitrógeno en el ganado lechero. Actualmente, existe una variedad de métodos analíticos disponibles para la determinación de MUN, la cual puede ser mediante métodos colorimétricos, enzimáticos o por espectroscopia infrarroja. Este último método es el más común pues permite estimar la concentración de urea en leche de forma simultánea con otros componentes lácteos en un periodo corto de tiempo. No obstante, relativa imprecisión es inherente a esta metodología pues en algunos casos los valores de MUN pueden ser subestimados o sobreestimados. Esto debido a la interferencia de otros componentes lácteos capaces de absorber luz a la longitud de onda de la urea. El principal componente que interfiere es la grasa láctea, la cual es muy sensible a la variación pues tanto su contenido como la composición de ácidos grasos pueden ser afectados por el impacto de la manipulación dietética sobre el metabolismo microbial en el rumen. La asociación entre la suplementación con dietas depresoras de grasa láctea y la concentración de MUN es contradictoria, pero en apariencia no existe un efecto directo sobre el MUN cuando este es determinado por métodos enzimáticos. En la actualidad no existen estudios que hayan investigado la asociación de este tipo de dietas con el MUN estimado por medio de espectroscopía infrarroja. Sin embargo, la presente revisión sugiere que este método de determinación subestimaría la concentración de MUN con este tipo de dietas.

Palabras clave: ganado lechero, nitrógeno ureico en leche, espectroscopía infrarroja, ácidos grasos poliinsaturados, grasa láctea.

\footnotetext{
${ }^{1}$ Animal Nutrition Group, Wageningen University. Wageningen, the Netherlands. Autor para correspondencia: jeffry.sanchezsalas@gmail.com Recibido: 23 de mayo 2016 Aceptado: 8 de agosto 2016
} 


\section{ABSTRACT}

Milk urea nitrogen: Importance, determination and relationship with other milk components. Milk urea concentration is proportional to the amount of urea in the blood. Therefore the analysis of milk urea nitrogen (MUN) has been adopted as a tool to monitor nitrogen utilization efficiency in dairy cattle. Currently, a variety of analytical methods is available for MUN determination, which can be done by colorimetric, enzymatic or infrared spectroscopy methods. The latter is the most common since it allows estimating milk urea concentration simultaneously with other milk components in a short period of time. However, relative inaccuracy is inherent to this methodology as MUN values could be underestimated or overestimated in some cases. This is due to the interference of other milk components capable of absorbing light at the wavelength of urea. The main interfering component is milk fat, which is highly variable since its content and fatty acids composition can be affected by the impact of diet manipulation on rumen microbial metabolism. The association between milk fat depression diets supplementation and MUN concentration is contradictory, but in appearance there is no direct effect on MUN when is determined by enzymatic methods. At present, there are no studies that have investigated the association between these diets and MUN estimated by means of infrared spectroscopy. However, this review suggests that this method would underestimate MUN concentration with these types of diets.

Keywords: dairy cattle, milk urea nitrogen, infrared spectroscopy, polyunsaturated fatty acids, milk fat. 


\section{INTRODUCCIÓN}

Es sabido que la concentración de urea en leche es proporcional a la cantidad de urea en la sangre (Jonker et al., 1998). Por esta razón, el análisis de nitrógeno ureico en leche (MUN, por sus siglas en inglés) ha sido adoptado como una herramienta para monitorear una adecuada sincronización de sustratos y una eficiente utilización del nitrógeno en el ganado lechero que contribuya a minimizar las pérdidas de este mineral sin afectar la producción de leche (Johnson y Young 2003; Wattiaux et al., 2005).

Actualmente, existe una variedad de métodos analíticos disponibles para la determinación de MUN. La instrumentación automática por espectroscopía infrarroja se ha convertido en la más común pues permite estimar la concentración de MUN de una forma rápida y económica (Godden et al., 2000). Sin embargo, los autores destacan que otros componentes lácteos son capaces de absorber cierta cantidad de luz a la longitud de onda de la urea que pueden conllevar a una subestimación o sobrestimación del MUN. Por ejemplo, algunos autores han establecido una asociación positiva entre la concentración de MUN y grasa láctea (Godden et al., 2000; Peterson et al., 2004).

Algunos estudios han demostrado que las dietas suplementadas con ácidos grasos poliinsaturados (PUFA) alteran tanto el contenido de grasa láctea como la composición de ácidos grasos debido a los efectos adversos sobre ciertos microorganismos del rumen (Bharatan et al., 2008; Boeckaert et al., 2008a; Klop et al., 2016). Por estas razones, el objetivo de la presente revisión es 1) investigar los factores asociados a la concentración de MUN y las implicaciones de su determinación por diferentes métodos, así como 2) establecer la relación entre las dietas suplementadas con PUFA y el contenido de MUN.

\section{Urea en leche como indicador de la utilización del nitrógeno en ganado lechero}

La urea es el metabolito final de los compuestos nitrogenados predominante en los mamíferos. Este compuesto es formado en el hígado y transportado principalmente por vía sanguínea hacia los riñones, donde es excretada en la orina (Francis et al., 2002). Una porción es difundida a la leche a través de las membranas celulares, por lo que la urea presente en la leche es proporcional a la cantidad de urea en la sangre (Jonker et al., 1998). Por esta razón, el análisis de MUN provee un medio para estimar la excreción urinaria de nitrógeno (Kohn et al., 2004; Wattiaux et al., 2005). 
Asimismo, se ha generado un considerable interés en utilizar el MUN como un indicador práctico para evaluar los programas nutricionales de los hatos lecheros. Este indicador puede ser utilizado para determinar la eficiencia de utilización del nitrógeno de las vacas lecheras e identificar la sobrealimentación o subalimentación de proteína en la dieta (Jonker et al., 1998, 2002). Esto debido a que la sobrealimentación de proteína ha mostrado tener un impacto negativo en la salud y fertilidad del ganado lechero, así como contribuir con la contaminación ambiental por nitrógeno (Gustafsson y Carlsson 1993; Yan et al., 2006). Por el contrario, la subalimentación de proteína puede también resultar en perjuicio de la fertilidad y baja producción de leche (Gustafsson y Carlsson 1993; Law et al., 2009).

Dadas estas asociaciones, su monitoreo desde un punto de vista práctico ayudaría a tomar decisiones nutricionales que prevengan una falla metabólica de las vacas (Hanuš et al., 2008) y a comprender su impacto sobre la economía de los productores lecheros y el ambiente (Johnson y Young, 2003). Por esta razón, normalmente se realizan controles de MUN tanto a nivel individual como a nivel grupal o de hato (tanque), cuyos valores se consideran normales dentro del rango 8 - $25 \mathrm{mg} / \mathrm{dL}$ (Inal et al., 2015) y 12 - $16 \mathrm{mg} / \mathrm{dL}$ (Nelson 1996), respectivamente. No obstante, debido a la considerable variabilidad entre los niveles de MUN entre vacas, se ha recomendado que estos valores sean interpretados a nivel grupal y no individual (Godden et al., 2001b).

\section{Factores asociados con la concentración de MUN}

A pesar de los rangos propuestos, es sabido que los resultados de MUN y su interpretación dependen además de una serie de factores a nivel de hato como la producción de leche promedio de la finca (Rajala-Schultz y Saville, 2003) o a nivel de vaca como la raza, el número de lactancia y la etapa de lactancia, así como el método de muestreo y la época del año (Godden et al., 2001ab; Wattiaux et al., 2005) tal como se muestra en el Cuadro 1. 
Cuadro 1. Concentración de MUN (mg/dL) asociada con el número de lactancia, mes de lactancia, época, y tipo de muestra reportada para diferentes niveles de producción y razas

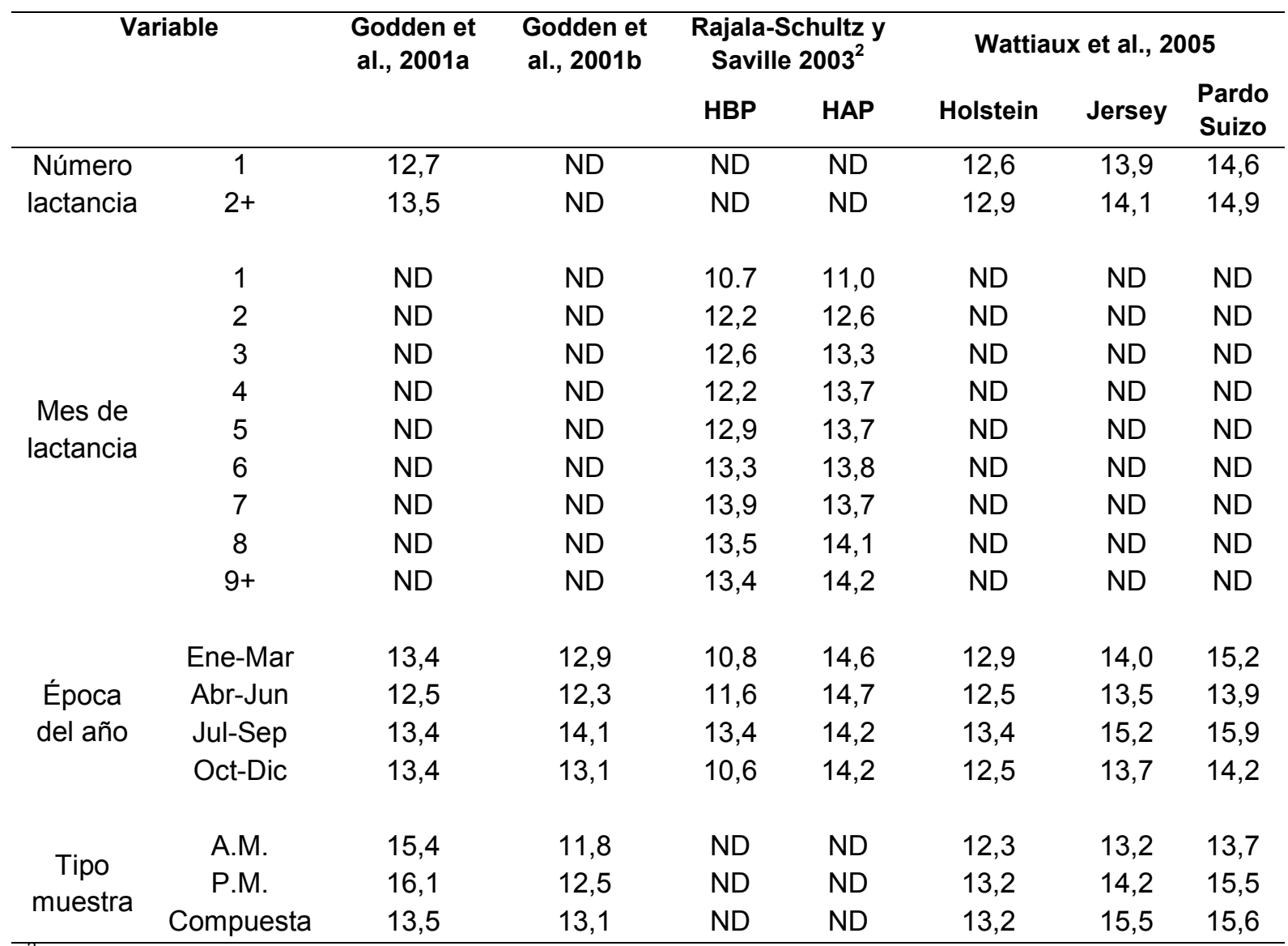

${ }^{2} \mathrm{HBP}=$ Hatos de baja producción definidos con una producción anual inferior a $7.258 \mathrm{~kg}, \mathrm{HAP}=$ Hatos de alta producción definidos con una producción anual mayor a $10.433 \mathrm{~kg}$. ND = No determinado.

Algunos estudios han evaluado la relación entre el MUN y variables productivas o nutricionales en hatos lecheros. Tanto Godden et al., (2001a) como Johnson y Young (2003) reportaron una relación positiva entre la concentración de MUN y la producción de leche. Godden et al., (2001b) reportaron una asociación positiva entre el MUN y la concentración de proteína cruda (PC), proteína degradable en rumen (PDR) y proteína no degradable en rumen (PNDR) contrario a la asociación negativa entre el MUN y la concentración de carbohidratos no fibrosos (CNF), así como con las relaciones entre estos nutrientes (CNF:PC, CNF:PDR, CNF:PNDR). 
Una explicación para la relación positiva entre el MUN y la producción de leche es que un mayor contenido de proteína en la dieta, también podría permitir una mayor producción de leche (Law et al., 2009). Esto sería debido a una combinación de factores que incluyen una mayor disponibilidad de aminoácidos para la síntesis de proteína láctea, mayor disponibilidad de energía a través de la desaminación de aminoácidos, mejor eficiencia de utilización de los nutrientes absorbidos o mayor consumo de materia seca (Oldham 1984; Law et al., 2009).

Sin embargo, Godden et al., (2001b) destacan que una relación negativa entre estas variables podría resultar del gasto de energía asociado con la conversión de excesos de amonio a urea que reduce la disponibilidad de energía para producción de leche. La asociación entre la utilización eficiente de nitrógeno y variables productivas recién descrita sería de utilidad para minimizar las pérdidas de nitrógeno manteniendo la producción de leche (Johnson y Young, 2003; Peterson et al., 2004).

\section{Métodos para la determinación de MUN}

Desde 2004, la Federación Internacional de Lechería (IDF, por sus siglas en inglés) especificó un método enzimático para la determinación del contenido de urea en leche mediante la medición de la diferencia de $\mathrm{pH}$ como el método de referencia ${ }^{1}$. En la actualidad, existe una variedad de métodos analíticos desarrollados para la determinación de urea en leche; Francis et al., (2002) describe varios de estos métodos, los utilizados con mayor frecuencia se detallan en el Cuadro 2.

\footnotetext{
${ }^{1}$ International Standard ISO 14637 | IDF 195.
} 
Cuadro 2. Características de la metodología actual para la determinación de urea en leche

\begin{tabular}{|c|c|c|c|}
\hline & Métodos colorimétricos & Métodos enzimáticos & Espectroscopía infrarroja \\
\hline Principio & $\begin{array}{l}\text { Esta técnica involucra la } \\
\text { formación de compuestos } \\
\text { rojos o anaranjados } \\
\text { producto de la reacción de } \\
\text { la urea y diacetilmonoxima, } \\
\text { esto debido a que la } \\
\text { hidrólisis ácida de este } \\
\text { último a diacetilo libera } \\
\text { hidroxilamina. El color es } \\
\text { estabilizado con la adición } \\
\text { de sales de hierro (III) y } \\
\text { tiosemicarbazida }\end{array}$ & $\begin{array}{l}\text { La determinación es basada } \\
\text { en la reacción Berthelot, en } \\
\text { la cual tras la aplicación de } \\
\text { ureasa el amonio liberado } \\
\text { reacciona con fenol y el ion } \\
\text { hipoclorito para producir un } \\
\text { color azul, particularmente } \\
\text { en la presencia de un } \\
\text { complejo nitrito pentaciano } \\
\text { ferroso formado con la } \\
\text { adición de nitroprusiato de } \\
\text { sodio }\end{array}$ & $\begin{array}{l}\text { Esta técnica expresa los } \\
\text { modos de vibración de los } \\
\text { enlaces covalentes en las } \\
\text { moléculas. Actualmente se } \\
\text { basa en el principio } \\
\text { Transformada de Fourier } \\
\text { (FTIR) que asiste en la } \\
\text { cuantificación de cualquier } \\
\text { componente que absorba } \\
\text { radiación infrarroja en la } \\
\text { muestra puesto que } \\
\text { determina un espectro } \\
\text { completo }\end{array}$ \\
\hline Ventajas & $\begin{array}{l}\text { Diacetilmonoximatiene alta } \\
\text { estabilidad y selectividad en } \\
\text { muchas matrices de } \\
\text { muestras y se ha aplicado a } \\
\text { la instrumentación de } \\
\text { análisis por inyección en } \\
\text { flujo (FIA) }\end{array}$ & $\begin{array}{l}\text { El método más preciso para } \\
\text { la determinación de MUN y } \\
\text { es reconocido como el } \\
\text { método de referencia por la } \\
\text { IDF }\end{array}$ & $\begin{array}{l}\text { Un espectro completo de la } \\
\text { muestra es brindado en un } \\
\text { corto periodo de tiempo, por } \\
\text { lo que permite analizar al } \\
\text { mismo tiempo otros } \\
\text { componentes como grasa, } \\
\text { proteína, lactosa y CCS }\end{array}$ \\
\hline Desventajas & $\begin{array}{l}\text { Impráctico en hatos } \\
\text { lecheros a larga escala } \\
\text { debido a la labor requerida } \\
\text { para el manejo de las } \\
\text { muestras, además de ser } \\
\text { lento y costoso }\end{array}$ & $\begin{array}{l}\text { Impráctico en hatos } \\
\text { lecheros a larga escala } \\
\text { debido a la labor requerida } \\
\text { para el manejo de las } \\
\text { muestras, además de ser } \\
\text { lento y costoso }\end{array}$ & $\begin{array}{l}\text { Análisis multivariados son } \\
\text { requeridos para la } \\
\text { estimación de urea por el } \\
\text { traslape de las bandas de } \\
\text { absorción de los otros } \\
\text { componentes }\end{array}$ \\
\hline \multirow[t]{4}{*}{$\begin{array}{l}\text { Instrumentación } \\
\text { rutinaria }\end{array}$} & \multirow{2}{*}{$\begin{array}{l}\text { Método } \\
1-4 \\
\text { paradimetilaminobenzaldehí } \\
\text { do (DMAB) }\end{array}$} & $\begin{array}{l}\text { Conductimetría Beckmann, } \\
\text { BUN Analyser }\end{array}$ & $\begin{array}{l}\text { Milkoscan (Foss Electric, } \\
\text { DK) }\end{array}$ \\
\hline & & $\begin{array}{l}\text { Diferencial } \mathrm{de} \quad \mathrm{pH} \\
\text { Eurochem, CL10; Hamilton, } \\
\text { E.F.A. }\end{array}$ & $\begin{array}{l}\text { 4000, FT } 120 \text { (FTIR), FT } \\
6000 \text { (FTIR) }\end{array}$ \\
\hline & \multirow{2}{*}{$\begin{array}{l}\text { Método } \\
\text { (DAM) }\end{array}$} & $\begin{array}{l}\text { Fotometría UV Flow } \\
\text { Injection Analysis (FIA) }\end{array}$ & Instruments) \\
\hline & & 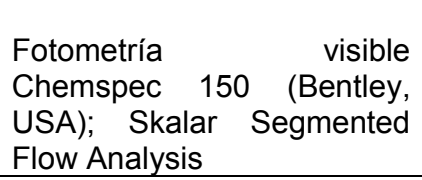 & FTIR Auto 400 \\
\hline
\end{tabular}

Fuentes: Andersen et al., 2002; Francis et al., 2012; International Committee for Animal Recording, 2016.

En resumen, la determinación de MUN puede realizarse obteniendo urea directamente o amonio como producto resultante de la reacción con ureasa; donde la cantidad de urea presente en la muestra es estimada midiendo el cambio de color en el espectrofotómetro 
o el diferencial de $\mathrm{pH}$, este último es reconocido como el método estándar. Asimismo, esta determinación puede realizarse a través de espectroscopía infrarroja basándose en la cantidad de luz absorbida a cierta longitud de onda (Arunvipas et al., 2003; Inal et al., 2015).

Sin embargo, algunos autores (Arunvipas 2003; Hanuš et al., 2008; Inal et al., 2015) consideran que la determinación enzimática por química húmeda es la técnica más precisa para detectar urea en leche, pues se categoriza como un método directo de medición. No obstante, Godden et al., (2000) destaca que a pesar de que esta tecnología ha estado disponible por mucho tiempo, ha sido impráctico medir la concentración de MUN en hatos lecheros a gran escala debido a la labor requerida para el manejo de las muestras, además de ser lenta y costosa.

\section{Implicaciones de la determinación de MUN por medio de espectroscopia infrarroja}

Bajo las consideraciones anteriores, la determinación de MUN basada en espectroscopía infrarroja, se ha convertido desde hace unos 25 años en un medio rápido y barato para estimar la concentración de urea en leche (Godden et al., 2001a). Numerosos y simultáneos análisis pueden ser conducidos en un periodo corto de tiempo pues el mismo instrumento puede ser utilizado para la determinación de grasa, proteína, lactosa y conteo de células somáticas (CCS), por lo que no se requiere un manejo separado de las muestras (Arunvipas et al., 2003; Inal et al., 2015).

El método es eficiente, pero resultados incorrectos pueden ser obtenidos en casos donde el número de muestras es bajo. Además, en algunos casos los valores de MUN pueden ser subestimados o sobrestimados (Inal et al., 2015). Esto puede ser atribuido a la interferencia de otros componentes lácteos conocidos por absorber cierta luz a la longitud de onda de la urea (1000 - $1700 \mathrm{~cm}^{-1}$; Nygaard et al., 1993). En este sentido, Hansen (1998) identificó en el espectro infrarrojo que la mayor parte de las contribuciones para la absorción de urea se sitúan en las regiones de 1161 y $1465 \mathrm{~cm}^{-1}$, siendo esta última banda la de principal absorción.

Precisamente, esta banda coincide con una de las bandas de absorción para grasa (1460 $\mathrm{cm}^{-1}$, enlace C-H) reportada por Hansen (1998), mientras que el rango propuesto 
concuerda con las regiones determinadas para la denominada grasa B (1680 - $1800 \mathrm{~cm}^{-1}$, enlace $C=0$ ) indicada por Lefier et al., (1996), así como la región mezclada que contiene información acerca de proteína y lactosa $\left(1000-1600 \mathrm{~cm}^{-1}\right)$ identificada por Andersen et al., (2002). Por esta razón, es necesaria la utilización de un algoritmo para ajustar la estimación de urea (Arunvipas et al., 2003), pues apenas $45-50 \%$ del valor es obtenido de la lectura óptica de la urea en la muestra mientras que la porción restante corresponde al ajuste matemático (Godden et al., 2000).

La relativa imprecisión de los resultados es inherente a la metodología infrarroja, algunas investigaciones se han realizado para establecer el nivel de concordancia entre los resultados obtenidos con diferentes métodos. Las concentraciones de MUN utilizando tanto el FOSS 4000 como el método estándar Eurochem CL10 no fueron diferentes de acuerdo con Godden et al., (2000) y Arunvipas et al., (2003), quienes reportaron un coeficiente de correlación de concordancia de 0,860 y 0,972; respectivamente. Kohn et al. (2004) demostraron que el Bentley, Skalar, FOSS4000 y FOSS 6000 suministraron valores similares de MUN en comparación con el Eurochem CL10.

A pesar que estos resultados indican que en general hubo un buen nivel de concordancia entre métodos, Kohn et al., (2004) determinaron que el método FOSS 4000 produjo la mayor desviación estándar en cuanto a la diferencia media (Dif. media = -0,21 mg/dL; DE $=2,51 \mathrm{mg} / \mathrm{dL}$ ). En una investigación similar, Peterson et al., (2004) reportaron que la menor variación en cuanto a la recuperación de urea añadida a la leche resultó para el método Eurochem CL10 (Rec. $=85,0 \%$; $E E=2,76 \%)$ mientras que la mayor fue para el FOSS 4000 (Rec. $=47,1 \%$; EE $=9,88 \%)$.

Las investigaciones realizadas por Kohn et al., (2004) y Peterson et al., (2004) demostraron que las mediciones de MUN obtenidas con el FOSS 4000 no fueron consistentes con otros equipos de medición y altamente variables. Asimismo, Peterson et al. (2004) reportaron que al utilizar el FOSS 6000 los niveles de recuperación fueron superiores o inferiores al 100\%, aspectos que sugieren un sesgo en la utilización de espectroscopía infrarroja que puede resultar en una subestimación o sobreestimación de la concentración de MUN. 
La determinación acertada de urea en leche es entorpecida por la opacidad y variación en el contenido de grasa de la matriz (Francis et al., 2002). Fueron Jonker et al., (1998) quienes predijeron que una variación en el contenido de grasa láctea de $\pm 0,5$ unidades porcentuales cambiaría la estimación de la concentración de MUN en $\pm 1,70 \mathrm{mg} / \mathrm{dL}$. Posteriores investigaciones han establecido una relación positiva (Godden et al., 2000; Godden et al., 2001b; Rajala-Schultz y Saville, 2003) o negativa (Godden et al., 2001a; Johnson y Young, 2003; Peterson et al., 2004) entre la concentración de MUN y el contenido de grasa láctea (Cuadro 3).

Cuadro 3. Relación lineal entre $\mathrm{MUN}(\mathrm{mg} / \mathrm{dL})$ y la composición láctea a nivel de vaca o hato reportado por diferentes autores

\begin{tabular}{|c|c|c|c|c|c|}
\hline Variable & Nivel & Método & $\begin{array}{l}\text { Pendiente } \\
\text { estimada }\end{array}$ & $\begin{array}{l}\text { Coeficiente } \\
\text { correlación }\end{array}$ & Valor $P$ \\
\hline \multicolumn{6}{|l|}{ Grasa láctea, \% } \\
\hline Godden et al., 2000 & Vaca & FOSS 4000 & & 0,41 & $<0,01$ \\
\hline Godden et al., 2001b & Hato & FOSS 4000 & 0,246 & & 0,0001 \\
\hline $\begin{array}{l}\text { Rajala-Schultz y Saville } \\
2003\end{array}$ & Hato & Skalar & 0,33 & & $<0,01$ \\
\hline Peterson et al., 2004 & Vaca & FOSS 6000 & $-0,679$ & & $<0,05$ \\
\hline \multicolumn{6}{|c|}{ CCS, unidades calificación lineal } \\
\hline Godden et al., 2000 & Vaca & FOSS 4000 & & $-0,011$ & $<0,0001$ \\
\hline $\begin{array}{l}\text { Rajala-Schultz y Saville } \\
2003\end{array}$ & Hato & Skalar & $-0,21$ & & $<0,01$ \\
\hline
\end{tabular}

Godden et al., (2000) identificaron hasta nueve componentes lácteos que pueden absorber cierta cantidad de luz a la longitud de onda de la urea, incluyendo proteína y CCS. En este sentido, algunas investigaciones han establecido una relación negativa entre la concentración de MUN y el contenido de proteína láctea y el CCS (Godden et al., 2001a; Johnson y Young, 2003; Rajala-Schultz y Saville, 2003).

A pesar de las circunstancias previamente descritas, algunos estudios (Godden et al., 2000; Godden et al., 2001ab) concluyen que cuando los resultados son interpretados a nivel de grupo o hato, la tecnología infrarroja para estimar la concentración de MUN sigue siendo una herramienta útil para monitorear la eficiencia de utilización del nitrógeno en hatos lecheros. 


\section{Alteraciones en el contenido de grasa láctea y su composición}

Es sabido que los valores de MUN obtenidos por métodos infrarrojos son estimados a través de ecuaciones de predicción; por lo tanto algunos estudios han reportado resultados controversiales en cuanto a la interferencia de otros componentes lácteos como la grasa (Godden et al., 2000; Godden et al., 2001b; Peterson et al., 2004). La grasa es el mayor componente energético de la leche, pero es muy sensible a la variación pues tanto su contenido como la composición de ácidos grasos pueden ser significativamente afectados por el impacto de la manipulación dietética sobre la población de bacterias del rumen y sus procesos microbiales (Bauman y Griinari, 2003). Uno de los ejemplos más notables fue reconocido por Davis y Brown (1970) como síndrome de baja grasa láctea.

En la actualidad es comúnmente conocido como depresión de grasa láctea y ocurre cuando al alimentar una dieta en particular se reduce marcadamente el contenido de grasa y se altera la composición de ácidos grasos de la leche (Bauman y Griinari, 2003). De acuerdo con Davis y Brown (1970) existen dos grupos de dietas capaces de inducir la depresión de grasa láctea, el primero incluye dietas que proveen altas cantidades de carbohidratos altamente digestibles y cantidades reducidas de componentes fibrosos mientras que el segundo grupo está representado por suplementos que contienen aceites polinsaturados (por ejemplo, aceites de origen vegetal o marino).

En este último caso, es conocido que los aceites y algas de origen marino inducen la depresión de grasa láctea aun cuando las dietas contengan niveles adecuados de fibra y están caracterizados por la presencia de dos PUFA: ácido eicosapentaenoico (C20:5) y ácido docosahexaenoico (DHA, C22:6). Las alteraciones en la actividad ruminal generalmente incluyen cambios en la proporción de ácidos grasos volátiles, específicamente una menor relación acetato:propionato, esto considerando que en los rumiantes cerca de la mitad de los ácidos grasos de la leche provienen de la síntesis de novo, para lo cual se utiliza acetato como mayor fuente de carbono (Bauman y Griinari, 2003).

Además, estas dietas causan alteraciones en la biohidrogenación ruminal que consiste en la saturación de ácidos grasos insaturados como el ácido linoleico y el ácido linolénico (Boeckaert et al., 2008b), siendo una característica clave la acumulación de 
intermediarios, particularmente ácidos grasos trans 18:1. De hecho, se asumía que un incremento en la depresión de grasa láctea era debido a la presencia del isómero trans-11 18:1, el cual es el mayor ácido graso transoctadecenoico presente en la grasa láctea (Bauman y Griinari, 2003).

Sin embargo, Griinari et al., (1998) descubrieron que la depresión de grasa láctea estaba asociada con un específico incremento en trans-10 18:1 en lugar de los isómeros trans 18:1 en general. Este ácido graso es formado a partir de la reducción del trans-10,cis-12 CLA en una ruta menor para la biohidrogenación del ácido linoleico. Al respecto, Kepler et al. (1966) demostraron que las bacterias ruminales Butyrivibrio fibrisolvens eran capaces de hidrogenar el trans-10,cis-12 CLA a trans-10 18:1 mientras que posteriormente Kim et al., (2002) comprobaron que la cepa Megasphaera elsdenii YJ-4 era capaz de producir trans-10, cis-12 CLA.

Por lo tanto, la teoría de la biohidrogenación propone que la depresión de grasa láctea inducida por este tipo de dietas es el resultado de una supresión directa de la síntesis de grasa láctea en la glándula mamaria en respuesta a ciertos ácidos grasos intermediarios formados durante la biohidrogenación e isomerización de PUFA, específicamente el trans10,cis-12 CLA (Bauman y Griinari, 2003; Weimer et al., 2015) aunque otros como cis10,trans-12 y trans-9,cis-11 también han mostrado tener efectos antilipogénicos (Boeckaert et al., 2008a).

Un ejemplo del particular efecto sobre el metabolismo microbial en el rumen previamente detallado ha sido mostrado por el ácido graso DHA. Diversas investigaciones han demostrado que la suplementación de vacas lecheras utilizando un producto comercial a base de algas con dosis de $25 \mathrm{~g}$ de DHA/día (Moate et al., 2013), $50 \mathrm{~g}$ de DHA/día (Klop et al., 2016) y $73 \mathrm{~g}$ de DHA/día (Boeckaert et al., 2008a) redujo la concentración de grasa láctea en 24,26 y $20 \%$ mientras que la producción de grasa láctea fue reducida en 17, 28 y $55 \%$, respectivamente.

Liu et al. (2011) reportaron que una infusión ruminal administrada a razón del 0,5\% de la dieta redujo significativamente la población de Fibrobacter succinogenes que podría resultar en una disminución de la degradación de celulosa. Asimismo, los autores observaron un incremento en el flujo de trans-10,cis-12 CLA, el cual se atribuye a un incremento en la población de Megasphaera elsdenni. Estos hallazgos son reflejo de que 
la suplementación dietética con PUFA como el DHA presenta un efecto inhibitorio sobre el proceso de biohidrogenación ruminal y se asocia a una limitación en la síntesis de grasa láctea.

\section{Relación entre la suplementación con PUFA y la concentración de MUN}

La asociación entre la suplementación con dietas depresoras de grasa láctea y la concentración de MUN es contradictoria. Johnson et al., (2002) reportaron que vacas suplementadas con niveles de inclusión crecientes de oleaginosas presentaron mayores valores de MUN debido en parte a una mayor solubilidad del nitrógeno en dichas dietas. No obstante, según los autores es común que las vacas alimentadas con raciones que contienen oleaginosas tengan valores mayores de MUN como resultado de una mayor absorción de nitrógeno a través de la pared ruminal.

En el estudio realizado por Chichlowski et al., (2005) la concentración de MUN fue significativamente menor cuando las vacas fueron suplementadas con semilla de canola molida a razón del 14\%, pero los autores destacan que esto pudo deberse a que la dieta ofrecida a estas vacas tenía un nivel similar de PC pero mayor de EN, respecto a la dieta control. Además, esto también podría deberse a la asociación entre la concentración de MUN y el consumo de materia seca descrita previamente, pues algunos estudios (Boeckaert et al., 2008a; Moate et al., 2013) han encontrado que la suplementación con DHA disminuyó el consumo de materia seca.

No obstante, la investigación conducida por Bharatan et al., (2008) que consideró la inclusión de aceite de pescado a razón del 0,5\% de la dieta no afectó la concentración de MUN. Recientemente, el estudio de Klop et al., (2016) tampoco mostró ningún efecto de la suplementación con DHA sobre la concentración de MUN. Todas estas investigaciones precedentes determinaron la concentración de MUN con métodos enzimáticos; sin embargo, en esta última los autores observaron que al realizar dicha determinación también con espectroscopía infrarroja, los valores fueron usualmente muy bajos en comparación con el método enzimático, encontrándose una diferencia media entre ambos de 7,3 mg/dL pero más acentuada conforme disminuyó el contenido de grasa láctea (Klop et al., 2016, datos no publicados). 


\section{CONSIDERACIONES FINALES}

En conclusión, el MUN es ampliamente aceptado como herramienta para evaluar la eficiencia de utilización del nitrógeno en ganado lechero, por conveniencia su estimación se realiza rutinariamente por métodos infrarrojos a pesar de la reconocida relación con otros componentes lácteos como la grasa. Diversos estudios han demostrado que dietas suplementadas con PUFA alteran tanto el contenido de grasa como la composición de ácidos grasos sin afectar directamente la concentración de MUN cuando se analiza por métodos enzimáticos. Para efectos de esta revisión, se consideraron algunas oleaginosas y grasas de origen marino como fuentes de PUFA, pero algunos otros ingredientes de uso común en la suplementación del ganado lechero como los destilados de maíz (DDGS), algunas grasas de sobrepaso e incluso los forrajes tiernos son importantes fuentes de PUFA. Actualmente, no existen estudios que hayan investigado la asociación de este tipo de dietas cuando el MUN es estimado por espectroscopía infrarroja, por lo tanto sus efectos son desconocidos. Sin embargo, las circunstancias previamente descritas sugieren que la espectroscopía infrarroja subestimaría la concentración de MUN en dietas con altos niveles de PUFA. 


\section{LITERATURA CITADA}

Andersen, S. K., P. W. Hansen, y H. V. Andersen. 2002. Vibrational spectroscopy in the analysis of dairy products and wine. In: J. M. Chalmers, P. R. Griffiths (Eds.), Handbook of vibrational spectroscopy (pp. 3672-3681). John Wiley \& Sons Ltd, West Sussex, UK.

Arunvipas, P., J. A. van Leeuwen, I. R. Dohoo, y G. P. Keefe.2003. Evaluation of the reliability and repeatability of automated milk urea nitrogen testing. The Canadian Journal of Veterinary Research 67: 60-63.

Bauman, D. E., y J. M. Griinari. 2003. Nutritional regulation of milk fat synthesis. Annu. Rev. Nutr. 23: 203-227.

Bharatan, M., D. J. Schingoethe, A. R. Hippen, K. F. Kalscheur, M. L. Gibson, y K. Karges. 2008. Conjugated linoleic acid increases in milk from cows fed condensed corn distillers solubles and fish oil. J. Dairy Sci. 91: 2796-2807.

Boeckaert, C., B. Vlaeminck, J. Dijkstra, A. Issa-Zacharia, T. van Nespen, W. van Straalen, y V. Fievez. 2008a. Effect of dietary starch or micro algae supplementation on rumen fermentation and milk fatty acid composition of dairy cows. J. Dairy Sci. 91: 4714-4727.

Boeckaert, C., B. Vlaeminck, V. Fievez, L. Maignien, J. Dijkstra, y N. Boon. $2008 b$. Accumulation of trans $\mathrm{C}_{18: 1}$ fatty acids in the rumen after dietary algal supplementation is associated with changes in the Butyrivibrio community. Appl. Environ. Microbiol. 74 (22): 6923-6930.

Chichlowski, M. W., J. W. Schroeder, C. S. Park, W. L. Keller, y D. E. Schimek. 2005. Altering the fatty acids in milk fat by including canola seed in dairy cattle diets. J. Dairy Sci. 88: 3084-3094.

Davis, C. L., y R. E. Brown. 1970. Low-fat milk syndrome. In: A. T. Phillipson (Ed.), Physiology of digestion and metabolism in the ruminant (pp. 545-565). Oriel, Newcastle upon Tyne, UK. 
Francis, P. S., S. W. Lewis, y K. F. Lim. 2002. Analytical methodology for the determination of urea: current practice and future trends. TrAC Trends in Analytical Chemistry 21 (5): 389-400.

Godden, S. M., K. D. Lissemore, D. F. Kelton, J. H. Lumsden, K. E. Leslie, y J. S. Walton. 2000. Analytic validation of an infrared milk urea assay and effects of sample acquisition factors on milk urea results. J. Dairy Sci. 83: 435-442.

Godden, S. M., K. D. Lissemore, D. F. Kelton, K. E. Leslie, J. S. Walton, y J. H. Lumsden. 2001a. Factors associated with milk urea concentrations in Ontario dairy cows. J. Dairy Sci. 84: 107-114.

Godden, S. M., K. D. Lissemore, D. F. Kelton, K. E. Leslie, J. S. Walton, y J. H. Lumsden. 2001b. Relationships between milk urea concentrations and nutritional management, production, and economic variables in Ontario dairy herds. J. Dairy Sci. 84: 1128-1139.

Griinari, J. M., D. A. Dwyer, M. A. McGuire, D. E. Bauman, D. L. Palmquist, y K. V. V. Nurmela. 1998. Trans-octadecenoic acids and milk fat depression in lactating dairy cows. J. Dairy Sci. 81: 1251-1261.

Gustafsson, A. H., y J. Carlsson. 1993. Effects of silage quality, protein evaluation systems and milk urea content on milk yield and reproduction in dairy cows. Livest. Prod. Sci. 37: 91-105.

Hansen, P. W. 1998. Urea determination in milk using Fourier transform infrared spectroscopy and multivariate calibration. Milchwissenschaft 53: 251-255.

Hanuš, O., P. Hering, J. Frelich, M. Jílek, V. Genčurová, y R. Jedelská. 2008. Reliability of results of milk urea analysis by various methods using artificial milk control samples. Czech J. Anim. Sci. 53 (4): 152-161.

Inal, F., E. Gürbüz, B. Coşkun, y M. S. Alataş. 2015. A comparison of different analysis methods for milk urea nitrogen. Kafkas Univ. Vet.Fak.Derg. 21 (5): 767-772.

International Committee for Animal Recording. 2016. International agreement of recording practices. ICAR. Rome, Italy. 675 p. 
Johnson, K. A., R. L. Kincaid, H. H. Westberg, C. T. Gaskins, B. K. Lamb, y J. D. Cronrath. 2002. The effect of oilseeds in diets of lactating cows on milk production and methane emissions. J. Dairy Sci. 85: 1509-1515.

Johnson, R. G., y A. J. Young. 2003. The association between milk urea nitrogen and DHI production variables in western commercial dairy herds. J. Dairy Sci. 86: 30083015.

Jonker, J. S., R. A. Kohn, y J. High. 2002. Use of milk urea nitrogen to improve dairy cow diets. J. Dairy Sci. 85: 939-946.

Jonker, J. S., R. A. Kohn, y R. A. Erdman. 1998. Using milk urea nitrogen to predict nitrogen excretion and utilization efficiency in lactating dairy cows. J. Dairy Sci. 81: 2681-2692.

Kepler, C. R., K. P. Hirons, J. J. McNeill, y S. B. Tove. 1966. Intermediates and products of the biohydrogenation of linoleic acid by Butyrivibriofibrisolvens. J. Biol. Chem. 241: 1350-1354.

Kim, Y. J., R. H. Liu, J. L. Rychlik, y J. B. Russell. 2002. The enrichment of a ruminal bacterium (Megasphaeraelsdenii YJ-4) that produces the trans-10,cis-12 isomer of conjugated linoleic acid. J. Appl. Microbiol. 92: 976-982.

Klop, G., B. Hatew, A. Bannink, y J. Dijkstra. 2016. Feeding nitrate and docosahexaenoic acid affects enteric methane production and milk fatty acid composition in lactating dairy cows. J. Dairy Sci. 99: 1161-1172.

Kohn, R. A., K. R. French, y E. Russek-Cohen. 2004. A comparison of instruments and laboratories used to measure milk urea nitrogen in bulk-tank milk samples. J. Dairy Sci. 87: 1848-1853.

Law, R. A., F. J. Young, D. C. Patterson, D. J. Kilpatrick, A. R. G. Wylie, y C. S. Mayne. 2009. Effect of dietary protein content on animal production and blood metabolites of dairy cows during lactation. J. Dairy Sci. 92: 1001-1012.

Lefier, D., R. Grappin, y S. Pochet. 1996. Determination of fat, protein, and lactose in raw milk by Fourier transform infrared spectroscopy and by analysis with a conventional filter-based milk analyzer. J. AOAC Int. 79 (3): 711-717. 
Liu, S. J., D. P. Bu, J. Q. Wang, P. Sun, H. Y. Wei, L. Y. Zhou, y Z. T. Yu. 2011. Effect of ruminal pulse dose of polyunsaturated fatty acids on ruminal microbial populations and duodenal flow and milk profiles of fatty acids. J. Dairy Sci. 94: 2977-2985.

Moate, P. J., S. R. O. Williams, M. C. Hannah, R. J. Eckard, M. J. Auldist, B. E. Ribaux, J. L. Jacobs, y W. J. Wales. 2013. Effects of feeding algal meal high in docosahexaenoic acid on feed intake, milk production, and methane emissions in dairy cows. J. Dairy Sci. 96: 3177-3188.

Nelson, A. 1996.Practical applications of MUN analyses.Bovine Pract. 29: 85-95.

Nygaard, G. L., B. T. Lapp, y N. B. Arnvidarson. 1993. Method of determining urea in milk. A/S Foss Electric, Hillerod, Denmark.Assignee. US Pat. No. 5252829.

Oldham, J. D. 1984. Protein-energy interrelationships in dairy cows. J. Dairy Sci. 67: 10901114.

Peterson, A. B., K. R. French, E. Russek-Cohen, y R. A. Kohn. 2004. Comparison of analytical methods and the influence of milk components on milk urea nitrogen recovery. J. Dairy Sci. 87: 1747-1750.

Rajala-Schultz, P. J., y W. J. A. Saville. 2003. Sources of variation in milk urea nitrogen in Ohio dairy herds. J. Dairy Sci. 86: 1653-1661.

Wattiaux, M. A., E. V. Nordheim, y P. Crump. 2005. Statistical evaluation of factors and interactions affecting dairy herd improvement milk urea nitrogen in commercial Midwest dairy herds. J. DairySci. 88: 3020-3035.

Weimer, P. J., L. Da Silva Cabral, y F. Cacite. 2015. Effects of ruminal dosing of Holstein cows with Megasphaeraelsdenni on milk fat production, ruminal chemistry, and bacterial strain persistence. J. Dairy Sci. 98: 8078-8092.

Yan, T., J. P. Frost, R. E. Agnew, R. C. Binnie, y C. S. Mayne. 2006. Relationships among manure nitrogen output and dietary and animal factors in lactating dairy cows. J. Dairy Sci. 89: 3981-3991. 